\title{
AI Proposition for Crypt Information Management with Maximized EM Modelling
}

\author{
Sakshi Jolly, Neha Gupta,
}

\begin{abstract}
There are a few circumstances where we utilize cutting edge innovations to recognize another element from the information we have. Regardless of whether it might be finished data or halfway data we attempt to recognize the new thing from the information. Mysterious information is such we have to concentrate on estimating the situations of achievement rate with this sort of enigmatic information which resembles a futile information. Enigmatic esteem resembles a pointless information which resembled an old information. We have to refine that information which isn't certified at that time. For instance consider age in an informational index as the enigmatic information since when that informational index was made that client might be with some age and after such a significant number of years still the age will be continue as before in the dataset with no update. This sort of data can be handled utilizing the grouping instrument which can be distinguished dependent on the data we accumulated from the store. The usefulness referenced in this article is to quantify the enigmatic information with the AI and approving the model dependent on the exactness we scored with the present information accessible. The total article talks about the activities we perform to accomplish the exactness of the model with various grouping systems.
\end{abstract}

Keywords: Machine learning, Predictions, Modelling, Samples, cryptic data

\section{INTRODUCTION}

AI models are being utilized for the better fate of the humankind to streamline the way toward making and learning the things. As we cause the information or we to get the information we don't fret over the truth of the information we have. The truth of the data we have is the certifiable data we have must be helpful for the preparing. Not all the data we accumulate must be valuable for the demonstrating an expectation work yet some piece of data must be controlled at whatever point we require. Here comes the image of grave information which is a pointless data and this can't be associated with the ongoing data and the data we have may contain the examples which are unimportant to the displaying yet need to kup those as we go past our idea. What the significance of going past the considerations. The primary denounced thing here is the information we catch must be not garbage one. The garbage data can have the not refreshed data time to time and this is a sepulcher information. For instance consider any sort of data we have must be a real one and the data must be up to date.

Revised Manuscript Received on December 30, 2019.

* Correspondence Author

Sakshi Jolly, Research Scholar, Faculty of Computer Applications Department, MRIU, Faridabad (India)

Dr. Neha Gupta, Associate Professor, Faculty of Computer Applications Department, MRIIRS, Faridabad (India)

(C) The Authors. Published by Blue Eyes Intelligence Engineering and Sciences Publication (BEIESP). This is an open access article under the CC BY-NC-ND license (http://creativecommons.org/licenses/by-nc-nd/4.0/)
For an example consider information of a worker who has participated in an association hardly any years prior and after certain years he was turned into the CEO of the association and the data we need to consider is how he was thinking towards the activities and the tasks which can be anticipated for the future to outperform the other competitors.[1-4] This sort of related data can contain the garbage content dependent on which we need to foresee whether age can be an element to be considered for the expectation or not. In genuine the age must be known. Since to distinguish at which he found a way to get achievement in his life and profession. These are the little things which lead to the greater perspective on the issue. The issue here is the age can't be refreshed time to time and this can cause a blunder in the displaying and examining the data. [5-7]

Lets consider another case of distinguishing an example of instruction framework forecast and making the schedule or learning design dynamic to the understudies dependent on their individual execution. The individual execution of the understudy characterizes their future and the idea here is to distinguish the example to make the schedule or the learning segment dynamic to the understudy dependent on their exhibition. This can improve a group dependent on the bunch we can delineate class of the understudies and for that classification we can plan to make the dynamic move of the data. AI expectation models and grouping models like $\mathrm{K}$ Means has performed with some amazing information and the outcomes are some range fulfilled yet not totally. The explanation for is the grave information which is by all accounts little however assumes a pivotal job in the displaying.

The tomb information here is the score of the understudy which changes time to time with subject to subject. On the off chance that the understudy is solid in coding it doesn't imply that he can break and finish the hackethon. On the off chance that the understudy is dull in clarifying the idea, it doesn't imply that he can't finish the course work. In some purpose of time the belief system of the understudy will change now and again and the idea here is to distinguish the attitude of the understudy as for the work he was doing in that time.

For example we have a sample explanation on the things to be done follows. Cryptic value is the old data or not exact information. Example we get 2012 student details but presently age is old data so that data is cryptic values. "Dirty data" is such a pervasive problem in every company, in every industry! But we have lived with "dirty data" for decades, so why is it such a problem now? Is it because we promise to deliver data warehouses with "clean, integrated, historical data in a short time frame for low cost". In our project we are using forest dataset[8-9]. Link https://figshare.com/articles/Dataset_1_Validated_natural_a nd_cryptic_mRNA_splicing_mutations/7812323/1) 
Here cryptic data is meter information it is not exact data for our calculation so we changed feet from meter.

Proposed calculation to attain data trait:

$1 \mathrm{~m}=(1 / 0.3048) \mathrm{ft}=3.28084 \mathrm{ft}$.

The dataset we provided is the public dataset which gives the information about the mRNA concept of slicing the information in to different part. Here we have the ideology of implementing some of the machine learning models like $\mathrm{K}$-Means and the primary explanation is to conquered the impact we are getting with displaying with the grave information and the examples we considered must not be having the sepulcher information which gives the high fluctuation with the exactness.

Figure 1 shows the AI the CNN and the AI usage for the example issue of the element recognizable proof from an example of data as sight and sound. This data isolates the picture into fragments and procedure. For this situation, we can get the sepulcher information. With the goal that we have to process that first.

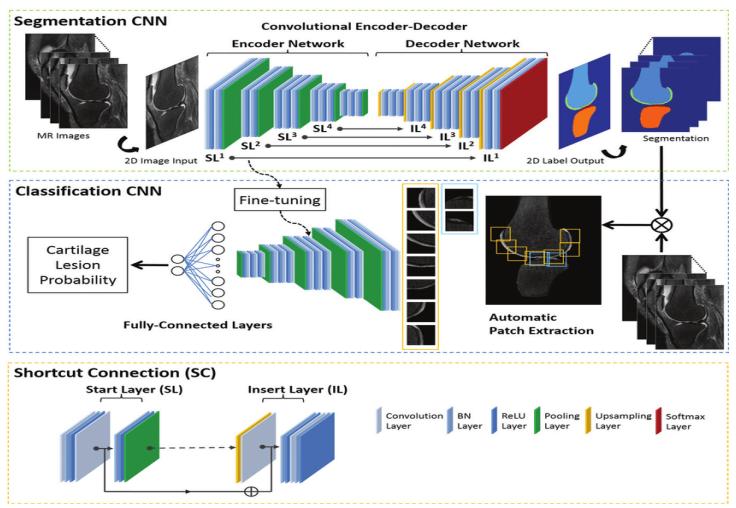

Figure 1: CNN for identifying crypt data From an image classification

The parallel piece of the area manages the current framework and the proposed methodologies. The design have the outcomes with the example data and will be exhibited in this article later areas lastly finish up.

\section{EXISTING SYSTEM}

The EM model is for the emphasis of the total method of AI and the gauge which we are thinking about will catch up the missing information and the data we assemble will be founded on every cycle result. The yield of one cycle will make the contribution for the following emphasis. At the point when we arrive at the desire in the outcome then we measure the probability of the events and the M-step augments the information is the thing which we get in the wake of examining and the demonstrating. We have to outline data utilizing $\mathrm{M}$ - step and the probability capacity will transform into greatest under the suspicions of missing information[11]. The assessed an incentive from the E-Step is considered for the assembly. $L(\vartheta \mid Y)=$ $\log \prod_{v=1}^{V} q\left(y_{n} \mid \vartheta\right)=\sum_{v=1}^{V} \log \sum_{s=1}^{S} \alpha_{s} g_{s}\left(y_{v} ; \theta_{s}\right)$

For then again model consider the calculation with the example execution of the missing data and the equation and the method for the current framework is just a single way. The fundamental strategy drives the recognizing the missing data and the missing data prompts the way toward mapping with the present information. The watched Figure 2 and 3 shows the EM bunching method under various cycles of procedure.

EM algorithm (iteration: 24)

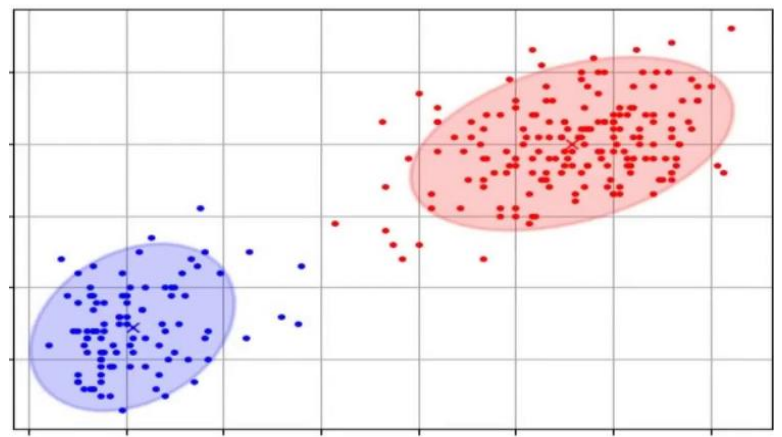

Figure 2: EM Clustering with the crypt data at iteration 24

Even at iteration 24 the information with the crypt data has not formed the model in the right path and we have some of the instances where we fail to map the correct information with the data.

EM algorithm (iteration: 36)

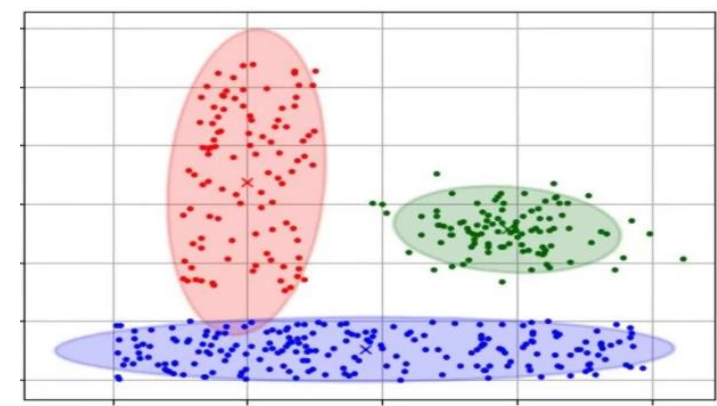

Figure 3: EM Modelling at iteration 36

In this situation in figure 3 the examples are additionally partitioned into various bunches dependent on the probability. Despite the fact that the groups are shaped this took long procedure to fix it. The calculations for the current framework is referenced as pursues

\section{M-step:}

Input: data, $\mathrm{X}, \alpha, \sigma$, l_clust

Output: $\alpha, \sigma$, prior

1. $\quad 1 \leftarrow$ data

2. for $\mathrm{T}$ in l_clust do

3. $\quad$ temp $1 \leftarrow$ data $-\alpha[\mathrm{T}]^{*} \mathrm{X}$

4. $\quad$ temp $2 \leftarrow$ data- $\alpha[\mathrm{T}] . \mathrm{X}[\mathrm{T}]$

5. $\quad$ set $\mathrm{a} \leftarrow$ normalize[a]

6. $\mathrm{T}=$ temp1+temp2

Return $\mathrm{T}$.

There is a difference in modeling with EM in the existing and proposed system we have increased the maximization content and that will be discussed in the next section.

\section{PROPOSED SYSTEM}

The proposed design comprises of the amplifying the MStep and distinguishing the missing qualities and the missing qualities can be utilized further for the check of the tomb data. This sort of data can be useful for recognizing the rightness of the model.

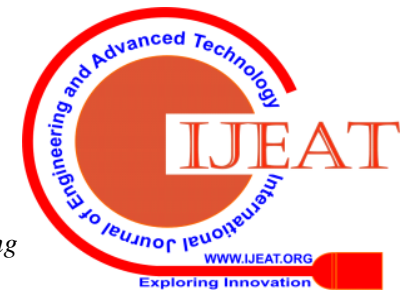


The current proposed model deals with a portion of the intriguing AI models like Gaussian model and the EM model was reached out with the most extreme M-Step as for the missing information in E-Step. [12]. To plot the data we have to have the capacity which figures the thickness. The underneath organization will manage the thickness work.

$$
p_{j}(y)=V\left(y ; \mu_{j}, \sigma_{j}\right)=\frac{1}{\sqrt{2 \pi} \sigma_{j}} \exp \left(-\frac{\left(y-\mu_{j}\right)^{2}}{2 \sigma_{j}^{2}}\right)
$$

In the M-step the problem of maximizing the likelihood is solved, using below equation.

$$
\begin{aligned}
w_{j} & =\frac{1}{m e} \sum_{i=1}^{m e} l_{i j} \\
\sigma_{j}^{2} & =\frac{1}{m e w_{j}} \sum_{i=1}^{m e} l_{i j} y_{i} \\
\sigma_{j}^{2} & =\frac{1}{m e w_{j}} \sum_{i=1}^{m e} l_{i j}\left(y-\mu_{j}\right)^{2}, j=\overline{1, a}
\end{aligned}
$$

The algorithm for the proposed system is mentioned as follows.

Input:data,1_clust, $\mathrm{v}, \varepsilon, \mathrm{a}=$ dataset

\section{Output: $\alpha, \sigma$,prior}

1. $\mathrm{l}, \mathrm{a} \leftarrow$ Row and column count

2. $\alpha \leftarrow$ making empty arrays using cluster $\mathrm{X}$ column

3. $\sigma \leftarrow$ making empty arrays using cluster $\mathrm{X}$ column X column

4. for $\mathrm{T}$ in 1_clust

5. $\mathrm{mu}[\mathrm{T}] \leftarrow \operatorname{data}[\mathrm{T}]$

6. $\operatorname{sigma}[\mathrm{T}] \leftarrow \mathrm{a}$

7. prior $\leftarrow$ repeat(1.0/1_clust,1_clust) for each cluster one prior

8. for $\mathrm{i}$ in $\mathrm{v}$ do

9. $\quad \alpha$ _old $\leftarrow 1 * \alpha$

10. $\mathrm{W} \leftarrow \mathrm{E} \_$step $($data $, \mu \quad, \quad \sigma$,prior,1_clust) \#calling E-step

11. $\alpha, \sigma$, prior $\leftarrow \mathrm{M} \_$step(data, $\mathrm{X}, \alpha, \sigma, 1 \_$clust $)$ \# calling M-step

12. Convergence check:

13. temp $\leftarrow 0$

14. for j in l_clust

15. temp $\leftarrow$ temp $+\sqrt{(\alpha}[j]-\alpha \_$old $)^{2}$

16. temp $\leftarrow$ round(temp,4)

17. if temp greater than $\varepsilon$ then

18. break

19. Return $\alpha, \sigma$,prior

E-step:
Input: data, $\alpha, \sigma, 1$ clust
Output: X
1. $1 \leftarrow$ data
2. $\mathrm{X} \leftarrow 1$ l clust $/ \mathrm{n}$
3. for c in l_clust do
4. $\mathrm{X}[\mathrm{c}] \leftarrow$ multivariate $($ data, mean $=\alpha$ $[\mathrm{c}]$, covarience $=\sigma[\mathrm{c}]$, allow_singular $=\mathrm{T}$ rue * prior[c])
5. temp $2 \leftarrow \operatorname{sum}(\mathrm{X})$
6. $\mathrm{X} \leftarrow \mathrm{X} /$ temp2
7. Return $X$

M-step: Input: data, $\mathrm{X}, \alpha, \sigma, 1$ clust

Output: $\alpha, \sigma$, prior
1. $1 \leftarrow$ data
2. for $\mathrm{T}$ in l clust do
3. temp $1 \leftarrow$ data $-\alpha[\mathrm{T}]^{*} \mathrm{X}$
4. $\quad$ temp $2 \leftarrow$ data- $\alpha[\mathrm{T}] . \mathrm{X}[\mathrm{T}]$

\author{
5. $\alpha[\mathrm{T}] \leftarrow \operatorname{dot} \operatorname{product}($ temp1,temp2)/ \\ $\operatorname{sum}(\mathrm{X}[\mathrm{T}])$ \\ 6. $\alpha[\mathrm{T}] \leftarrow \operatorname{sum}($ data $* \mathrm{X}[\mathrm{T}]) /$ \\ $\operatorname{sum}(\mathrm{X}[\mathrm{T}])$ \\ 7. $\operatorname{prior} \leftarrow \operatorname{sum}(\mathrm{X}) / \mathrm{l}$ \\ 8. $\operatorname{Return} \alpha, \sigma$, prior
}

In our proposed methodology we have changed the expansion step and $\mathrm{M}$ step utilizing the stage 5 and 6 . This is the curiosity of our methodology contrasted and typical EM clustering.[13-15] Our proposed calculation we think about a blend of Gaussian, we will keep on considering the EMcalculation for an ordinary conveyance. It is utilized to group the precise estimation of the dataset. Utilizing stage 5 and 6 , the littler extent will diminish and the greater extent will increment in the following emphasis and afterward rivalry will happen. We dispose of those bunches (or set those extents to get zero) and afterward update the group number

\section{RESULTS}

The both cases we proposed EM modelling but the difference comes with the maximization of the information. The maximization of the content can help the information to map and process the information. This can be considered as the information we gathered and the process of understating the difference between the existing system and the proposed system we have the sample table which explains the process of managing the crypt data. These kinds of crypt information have to work and manage well when needed. Table 1 and the graph indicating with figure 4 explain the process.

Table 1: Comparison Chart

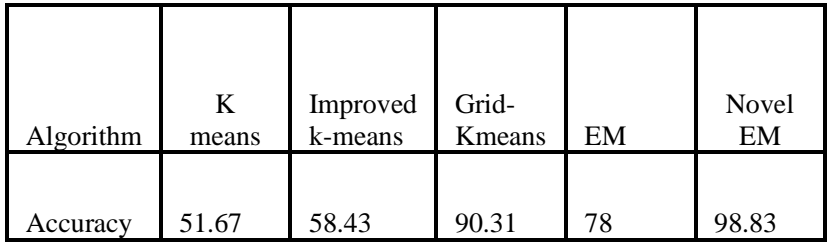

Table 2: Count of Clusters and Interations

\begin{tabular}{|l|l|l|l|}
\hline Algorithm & $\begin{array}{l}\text { No.of } \\
\text { Cluster }\end{array}$ & $\begin{array}{l}\text { No.of } \\
\text { Iteration }\end{array}$ & Time Taken \\
\hline K Means & 2 & 4 & 0.05 \\
\hline Hierarchical & 2 & 2 & 1.28 \\
\hline Density Base & 2 & 3 & 0.05 \\
\hline EM & 5 & 6 & 11.47 \\
\hline Proposed & 4 & 5 & 10.45 \\
\hline
\end{tabular}

The above table 2 demonstrates that what number of number of bunches are considered and the quantity of cycles dependent on the groups and the time taken to register. The example dataset we are thinking about is referenced underneath in table 3 and we can check diverse sort of grave data We have the example usage of the sepulcher information and the figure 5 and 6 arrangements with clarification of actualizing the tomb information and the information 


\section{AI Proposition for Crypt Information Management with Maximized EM Modelling}

we pursue here resembles a perplexity framework. The yield of the data will be as obvious positive and bogus positive. The exactness will be characterized dependent on the TP and FP.

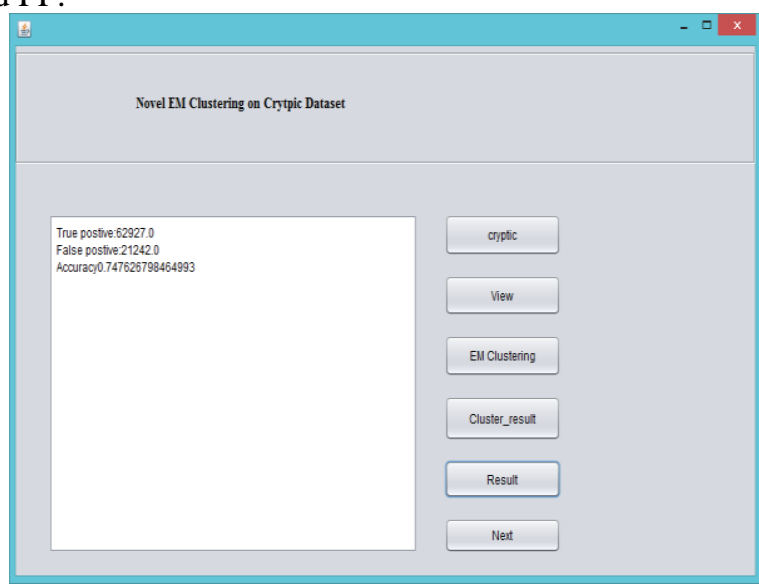

Figure 4: Result before applying the proposed EM model.

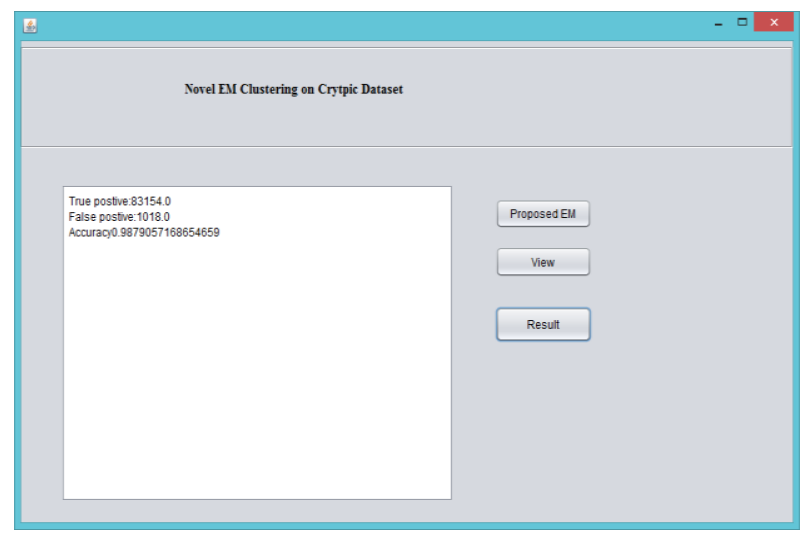

Figure 5: Result after applying the proposed EM model.

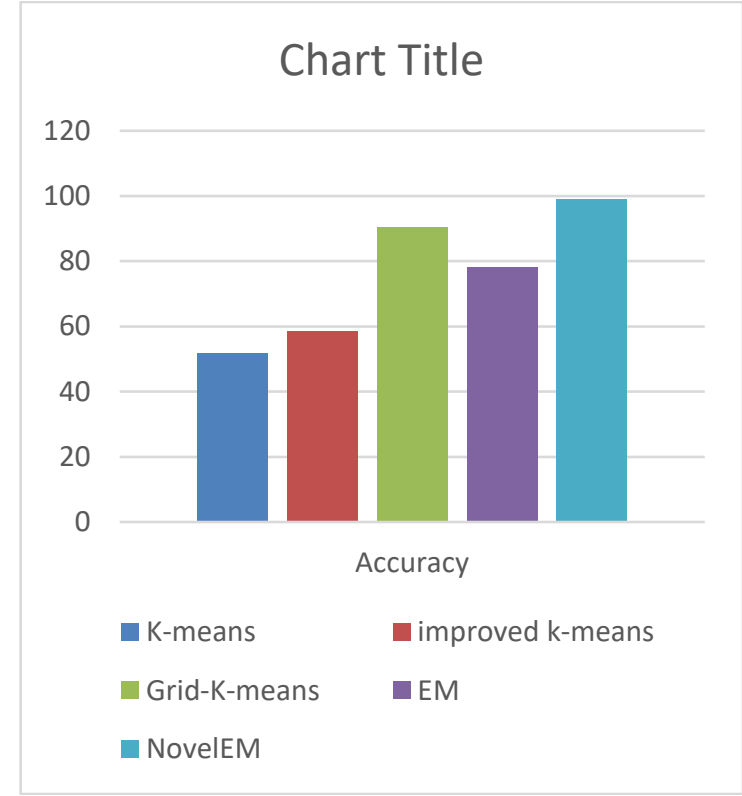

Figure 4: The comparisons are plotted in graph.

More than all the comparisons we have highest accuracy or the model with Novel EM which we proposed with maximizing the $\mathrm{M}$ step

\section{CONCLUSION}

Sepulcher issue and the tomb information is the pointless data which can be avoided from the model or we have to process the data in the correct manner. We process the data utilizing diverse sepulcher information displaying calculations and one of those is EM model. We handled in the EM model which was at that point referenced in the current framework and parcel work was done on the EM model to play out the better bunching of the information and here we prepared the data dependent on their recognition. That implies the client of the data or the maker of the data. We amplified the idea of $\mathrm{M}$ step and accomplished a similar arrangement in an ideal way with EM model by augmenting the $\mathrm{M}$ step.

\section{REFERENCES}

1. K. B. To and L. M. Napolitano, "Common complications in the critically ill patient," Surgical Clinics North Amer., vol. 92, no. 6, pp. 1519_1557, 2012.

2. C. M. Wollschlager and A. R. Conrad, "Common complications in critically ill patients," Disease-a-Month, vol. 34, no. 5, pp. 225_293, 1988.

3. S. V. Desai, T. J. Law, and D. M. Needham, "Long-term complications of critical care," Critical Care Med., vol. 39, no. 2, pp 371_379, 2011.

4. N. A. Halpern, S. M. Pastores, J. M. Oropello, and V. Kvetan, "Critical care medicine in the United States: Addressing the intensivist shortage and image of the specialty," Critical Care Med. vol. 41, no. 12, pp. 2754_2761, 2013.

5. A. E. W. Johnson, M. M. Ghassemi, S. Nemati, K. E. Niehaus, D. A. Clifton, and G. D. Clifford, "'Machine learning and decision support in critical care," Proc. IEEE, vol. 104, no. 2, pp. 444 466, Feb. 2016.

6. O. Badawi et al., "Making big data useful for health care: A summary of the inaugural MIT critical data conference," JMIR Med. Informat. vol. 2, no. 2, p. e22, 2014.

7. C. K. Reddy and C. C. Aggarwal, Healthcare Data Analytics, vol. 36 Boca Raton, FL, USA: CRC Press, 2015.

8. D. Gotz, H. Stavropoulos, J. Sun, and F. Wang, ' ICDA: A platform for intelligent care delivery analytics," in Proc. AMIA Annu. Symp. 2012, pp. 264273.

9. A. Perer and J. Sun, “Matrix_ow: Temporal network visual analytics to track symptom evolution during disease progression," in Proc. AMIA Annu. Symp., 2012, pp. 716_725.

10. Y. Mao,W. Chen, Y. Chen, C. Lu, M. Kollef, and T. Bailey, “'An integrated data mining approach to real-time clinical monitoring and deterioration warning," in Proc. 18th ACM SIGKDD Int. Conf. Knowl. Discovery Data Mining. 2012, pp. 1140_1148.

11. J. Wiens, E. Horvitz, and J. V. Guttag, "'Patient risk strati_cation for hospital-associated C. Diff as a time-series classi_cation task," in Proc. Adv. Neural Inf. Process. Syst., 2012, pp. 467475.

12. S. Saria, D. Koller, and A. Penn, "'Learning individual and population level traits from clinical temporal data," in Neural Inf. Process. Syst. (NIPS), Predictive Models Personalized Med. Workshop, 2010.

13. R. Dürichen, M. A. F. Pimentel, L. Clifton, A. Schweikard, and D. A Clifton, "Multitask Gaussian processes for multivariate physiological time-series analysis," IEEE Trans. Biomed. Eng., vol. 62, no. 1,pp 314_322, Jan. 2015.

14. M. Ghassemi et al., “'Amultivariate timeseries modeling approach to severity of illness assessment and forecasting in ICU with sparse, heterogeneous clinical data," in Proc. AAAI Conf. Artif. Intell., 2015, pp. 446_453.

15. I. Batal, H. Valizadegan, G. F. Cooper, and M. Hauskrecht, "A pattern mining approach for classifying multivariate temporal data," in Proc. IEEE Int. Conf. Bioinformatics Biomed. (BIBM), 2011, pp. 358365.

16. An overview on evocations of data quality at ETL stage, March 15, Available at: https://www.researchgate.net/publication/276922204 An overview on evocations of data quality at ETL stage.

17. Handling Mislaid/Missing Data to attain data trait, published in IJITEE, Available at ISSN: 2278-3075, Volume-8 Issue-12, October 2019, Page No. 4308-4311

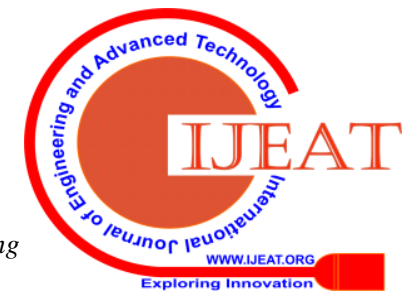


18. Higher Dimensional Data Access and Management with Improved Distance Metric Access for Higher Dimensional Non-Linear Data, published in IJRTE Volume-8 Issue-4, November 2019

19. Extemporizing The Data Trait, published in IJETT, published in Volume 58 Number 2 April issue.

20. Data quality outflow in cloud computing, published in INDIACom2016 at IEEE Xplore.

21. An Overview On Evocations Of Data Quality At Etl Stage, published in JNU conference in 2015.

\section{AUTHORS PROFILE}

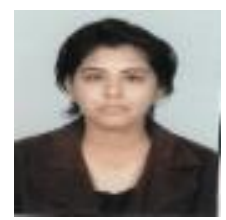

Ms. Sakshi Jolly, Research Scholar in Faculty of Computer Applications Department, MRIU, Faridabad, (India),total 4 years of teaching experience in the field of computers. She has authored 5 research papers in journals/conferences in the area of DQ.

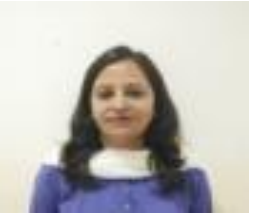

Dr. Neha Gupta, Associate Professor, Faculty of Computer Applications Department, MRIU, Faridabad (India) has total of $14+$ year of experience in teaching and research. She is a Life Member of ACM CSTA, Tech Republic and Professional Member of IEEE. She has authored and co-authored 34 research papers

SCI/SCOPUS/Peer Reviewed Journals (Scopus indexed) and IEEE/IET Conference proceedings in areas of Web Content Mining, Mobile Computing, and Cloud Computing. She has published books with publishers like IGI Global \& Pacific Book International and has also authored book chapters with Elsevier, CRC Press and IGI global USA. Her research interests include ICT in Rural Development, Web Content Mining, Cloud Computing, Data Mining and NoSQL Databases. She is a technical programme committee (TPC) member in various conferences across globe. She is an active reviewer for International Journal of Computer and Information Technology and in various IEEE Conferences around the world. 